COMMUNICATIONS IN

ANALYSIS AND GEOMETRY

Volume 14, Number 5, 1027-1035, 2006

\title{
Error estimates for discrete harmonic 1-forms over Riemann surfaces
}

\author{
WeI LuO
}

\begin{abstract}
We derive $L^{2}$ error estimates of computing harmonic or holomorphic 1-forms over a Riemann surface via finite element methods. Locally constant finite elements and first order approximations of the Riemann surface by triangulated meshes are considered. We use in the proof a Bochner type formula and a refined Poincaré inequality over a triangle of arbitrary shape.
\end{abstract}

\section{Introduction}

Surface matching and parametrization is a fundamental problem in 3-D computer graphics. In a series of papers [2], Gu and Yau applied the theory of Riemann surfaces to this problem. Given a Riemann surface $S$, they first compute harmonic 1-forms by minimizing the discretized energy functional of 1-forms in the same cohomology class. Then the space of holomorphic 1-forms on $S$ is constructed from harmonic 1-forms and the complex multiplication map $J$. From a holomorphic 1 -forms, one can construct a conformal coordinate over $S$, which could be used to match and compare similar surfaces. In their paper, the convergence of discrete holomorphic 1-forms to smooth ones is not addressed. Hence this paper provides a quantitative justification to this convergence (Theorem 3.6).

The proof goes as follows: we first use the Bochner formula on $S$ to estimate $\|\nabla \omega\|$ in terms of $\|\omega\|$ for a harmonic 1-form $\omega$. Then we prove a Poincaré inequality to estimate the error of projection onto finite element space in terms of norm of the gradient. Next we prove the main estimate of the error of projection of a smooth harmonic 1-form onto the space of discrete harmonic 1-forms. We finally give the error estimate of discrete holomorphic 1-forms and period matrix computation as a corollary.

\section{Notations}

Let $S$ be a closed smooth surface with metric $g . \quad J_{g}, \nabla_{g},\|\cdot\|_{g}$ respectively denote the almost complex structure, covariant derivative and $L^{2}$ norm 
associated to $g$. $N$ is a two-dimensional simplicial complex homeomorphic to $S$. If the lengths of edges of $N$ are given, $N$ naturally comes with a Euclidean metric on each face. Let $J, \nabla$ and $\|\cdot\|$ be the same notations as those with subscript $g$ but associated to the piecewise Euclidean metric. We assume that there exists a piecewise smooth homeomorphism $\phi: N \rightarrow S$ such that on each face $f$,

$$
\left|\phi^{*}(g)_{i j}-\delta_{i j}\right| \preceq M(f) l(f)^{2}, \quad\left|\nabla \phi^{*}(g)_{i j}\right| \preceq M(f) l(f), \quad M(f) l(f)^{2} \preceq 1,
$$

where $M(f)$ is some constant associated to the face $f, l(f)$ is the maximal length of edges of $f$. Here and throughout the paper, we use $\preceq$ to denote that the left-hand side is less than or equal to the right-hand side multiplied by some universal constant, independent of $N$ or $S$. Pulling back by $\phi$, we can regard a differential form or metric on $S$ as one on $N$. Without possibility of confusion, we omit the $\phi^{*}$ notation and identify forms or metric on $S$ and $N$ and denote them by the same notation. The following lemma is straightforward.

Lemma 2.1. With the assumption (2.1), let $\alpha$ be any tensor field; then on any face $f$, we have

$$
\begin{aligned}
\left.|| \alpha\right|_{g}-|\alpha| \mid & \preceq M(f) l(f)^{2}|\alpha|, \\
\left|J_{g}-J\right| & \preceq M(f) l(f)^{2} \\
\left|\nabla_{g} \alpha-\nabla \alpha\right| & \preceq M(f) l(f)|\alpha| .
\end{aligned}
$$

Example 2.2. When $S$ is a surface smoothly embedded in $\mathbb{R}^{3}, N$ is a polyhedral surface normally converging to $S$ (see [3]) and $\phi$ is the shortest distance map, a candidate of $M(f)$ is the maximum of principal curvature of $S$ on $\phi(f)$.

Definition 2.3. A discrete 1-form on $N$ is a piecewise continuous 1-form whose restriction onto each face is a constant 1-form. A discrete 1-form is closed if the evaluation of the 1-form on each edge is well- defined, i.e., evaluation by first restricting to either face adjacent to this edge will give the same value. A discrete 1-form is exact if it is the differential of a piecewise linear function on $N$. Closed discrete 1-forms differed by exact forms belong to the same cohomology class. A closed discrete 1-form is harmonic if it is the minimizer in its cohomology class of the energy functional $E(\omega)=$ $\|\omega\|^{2}=\int_{N}|\omega|^{2}$. Denote by $P_{H}$ the projection onto the space of discrete harmonic 1-forms under inner product associated to $E$. 
Let $\omega$ be a piecewise continuous 1 -form, one can project $\omega$ onto the space of discrete 1-forms under the inner product associated to $E$. Denote this projection by $P_{1}$. In a Euclidean coordinate associated to a face $f$, we have

$$
\left.P_{1}(\omega)\right|_{f}:=\left.\frac{1}{|f|} \int_{f} \omega\right|_{f}\left(\frac{\partial}{\partial x}\right) d x+\left.\frac{1}{|f|} \int_{f} \omega\right|_{f}\left(\frac{\partial}{\partial y}\right) d y
$$

Another way to produce a discrete 1 -form from closed $\omega$, denoted by $P_{2}(\omega)$, is by the condition:

$$
\int_{e}\left(\left.P_{2}(\omega)\right|_{f}-\left.\omega\right|_{f}\right)=0
$$

for any face $f$ and an edge $e$ on the boundary of $f$. Notice that $P_{2}(\omega)$ is also closed and is in the same cohomology class as $\omega$.

\section{Proof of the main results}

The following theorem estimates the error of the projections $P_{1}, P_{2}$ :

Theorem 3.1. Let $\Delta$ be a triangle with longest edge of length $l, \omega$ is a smooth closed 1 -form on $\Delta . P_{1}, P_{2}$ is defined as in (2.5) and (2.6), then

$$
\int_{\Delta}\left|\omega-P_{1}(\omega)\right|^{2} \leq \frac{2 l^{2}}{3} \int_{\Delta}|\nabla \omega|^{2}, \quad \int_{\Delta}\left|\omega-P_{2}(\omega)\right|^{2} \leq \frac{3 l^{2}}{2} \int_{\Delta}|\nabla \omega|^{2} .
$$

We need three lemmas first. The following is a refined Poincaré inequality for triangles (see also (7.45) in [1, p. 164]).

Lemma 3.2 (Poincaré inequality). If $u$ is a smooth function on a triangle $\Delta$, then

$$
\int_{\Delta}|u-\bar{u}|^{2} d x d y \leq \frac{2 l^{2}}{3} \int_{\Delta}|\nabla u|^{2} d x d y
$$

where $\bar{u}$ is the average of $u$ over $\Delta, l$ is the length of the longest edge of $\Delta$ and $\nabla u$ denotes the gradient of $u$. 
Proof.

$$
\begin{aligned}
\int_{\Delta}|u-\bar{u}|^{2}= & \int_{\Delta} u^{2}-2 u \bar{u}+\bar{u}^{2}=\int_{\Delta} u^{2}-\bar{u}^{2} \\
= & \int_{\Delta} u^{2}-\frac{1}{|\Delta|}\left(\int_{\Delta} u\right)^{2} 2\left(\int_{\Delta} 1 \int_{\Delta} u^{2}-\left(\int u\right)^{2}\right) \\
= & \int_{\Delta} \int_{\Delta}\left(u\left(x_{1}, y_{1}\right)-u\left(x_{2}, y_{2}\right)\right)^{2} d x_{1} d y_{1} d x_{2} d y_{2} \\
= & \int_{\Delta} \int_{\Delta}\left(\int _ { 0 } ^ { 1 } \frac { \partial } { \partial \lambda } u \left(x_{1}+\lambda\left(x_{2}-x_{1}\right), y_{1}\right.\right. \\
& \left.\left.+\lambda\left(y_{2}-y_{1}\right)\right) d \lambda\right)^{2} d x_{1} d y_{1} d x_{2} d y_{2} \\
\leq & \int_{\Delta} \int_{\Delta} \int_{0}^{1}\left(\left(x_{2}-x_{1}\right)^{2}+\left(y_{2}-y_{1}\right)^{2}\right) \\
& \cdot\left|\nabla u\left((1-\lambda)\left(x_{1}, y_{1}\right)+\lambda\left(x_{2}, y_{2}\right)\right)\right|^{2} d \lambda d x_{1} d y_{1} d x_{2} d y_{2} .
\end{aligned}
$$

Make the change of variables $\left(\lambda, x_{1}, y_{1}, x_{2}, y_{2}\right) \rightarrow\left(x_{0}, y_{0}, s, t, \theta\right)$ :

$$
\begin{gathered}
\left(x_{1}, y_{1}\right)=\left(x_{0}, y_{0}\right)+s(\cos \theta, \sin \theta), \\
\left(x_{2}, y_{2}\right)=\left(x_{0}, y_{0}\right)-t(\cos \theta, \sin \theta), \quad \lambda=\frac{s}{s+t} .
\end{gathered}
$$

Direct computation shows that the Jacobian of this change of variables is 1 . Write

$$
M=\max _{\left(x_{0}, y_{0}\right) \in \Delta} \int_{0}^{2 \pi} \int_{0}^{r_{1}} \int_{0}^{r_{2}}(s+t)^{2} d s d t d \theta
$$

where $r_{1}, r_{2}$ are the distance to $\left(x_{0}, y_{0}\right)$ from the intersection points of line $\left(x_{0}, y_{0}\right)+s(\cos \theta, \sin \theta), s \in \mathbb{R}$ with $\partial \Delta$. We then have

$$
|\Delta| \int_{\Delta} u^{2}-\left(\int_{\Delta} u\right)^{2} \leq \frac{M}{2} \int_{\Delta}|\nabla u|^{2} .
$$

On the other hand

$$
\int_{0}^{r_{1}} \int_{0}^{r_{2}}(s+t)^{2} d s d t=\frac{r_{2}^{3} r_{1}}{3}+\frac{r_{1}^{2} r_{2}^{2}}{2}+\frac{r_{2} r_{1}^{3}}{3} \leq \frac{7}{96}\left(r_{1}+r_{2}\right)^{4}
$$

Suppose the longest edge of $\Delta$ is $l$, the height on this edge is $h$, take coordinate $(x, y)$ such that the longest edge is on $x$-axis. Then for $\theta \leq \arctan (h / l)$, 


$$
\begin{aligned}
r_{1}+r_{2}<l . \text { For } \pi / 2 \geq \theta & \geq \arctan (h / l), r_{1}+r_{2} \leq \frac{h}{\sin \theta} \\
\int_{0}^{\arctan h / l} l^{4} d \theta & <l^{4} \cdot \frac{h}{l}=l^{3} h \\
\int_{\arctan h / l}^{\pi / 2}\left(\frac{h}{\sin \theta}\right)^{4} d \theta & =\int_{h / l}^{\infty} h^{4}\left(1+\frac{1}{k^{2}}\right)^{2} d \arctan k \\
& =\int_{h / l}^{\infty} h^{4}\left(k^{-4}+k^{-2}\right) d k=\frac{l^{3} h}{3}+l h^{3} \leq \frac{13}{12} l^{3} h
\end{aligned}
$$

where the last step is because when $l$ is the longest edge, $h<\frac{\sqrt{3}}{2} l$. Collectively, we have

$$
M \leq \frac{7}{96} \cdot 4 \cdot\left(1+\frac{13}{12}\right) l^{3} h<\frac{2}{3} l^{3} h
$$

plug this into Equation (3.3), the lemma is then proved.

Lemma 3.3. Let $u$ be a $C^{1}$ function on a triangle $\triangle A B C$ with maximal length of edges $l$. Then, we have

$$
\left|\frac{1}{|\Delta|} \int_{\Delta} u-\frac{1}{|B C|} \int_{B C} u\right|^{2} \leq \frac{l^{2}}{8|\Delta|} \int_{\Delta}|d u|^{2} .
$$

Proof. Take coordinate such that $A=\left(x_{A}, y_{A}\right), B=(0,0), C=\left(x_{c}, 0\right)$, and then the change of variable

$$
(x, y) \rightarrow\left((1-t) s x_{C}+t x_{A}, t y_{A}\right)
$$

Direct computations show that

$$
\operatorname{det}\left(\frac{\partial(x, y)}{\partial(s, t)}\right)=(1-t) x_{C} y_{A}, \quad|\Delta|=\frac{x_{C} y_{A}}{2}, \frac{\partial}{\partial t}=\left(x_{A}-s x_{C}\right) \frac{\partial}{\partial x}+y_{A} \frac{\partial}{\partial y} \text {. }
$$

The LHS of (3.5) is

$$
\begin{aligned}
& =\left(\int_{0}^{1} \int_{0}^{1} u(s, t) 2(1-t) d s d t-\int_{0}^{1} u(s, 0) d s\right)^{2} \\
& =\left(\int_{0}^{1} \int_{0}^{1}(u(s, t)-u(s, 0)) 2(1-t) d t d s\right)^{2} \\
& =\left(\int_{0}^{1} \int_{0}^{1} \int_{0}^{t} u_{\tau}(s, \tau) 2(1-t) d \tau d t d s\right)^{2}
\end{aligned}
$$




$$
\begin{aligned}
= & \left(\int_{0}^{1} \int_{0}^{1} u_{\tau}(s, \tau)(1-\tau)^{2} d \tau d s\right)^{2} \\
\leq & \left(\int_{0}^{1} \int_{0}^{1}|d u|^{2}(1-\tau) x_{C} y_{A} d \tau d s\right) \\
& \cdot \int_{0}^{1} \int_{0}^{1}\left(\left(x_{A}-s x_{C}\right)^{2}+y_{A}^{2}\right)(1-\tau)^{3}\left(x_{C} y_{A}\right)^{-1} d \tau d s \\
\leq & \text { RHS. }
\end{aligned}
$$

The proof of the following lemma is a straightforward computation.

Lemma 3.4. Given unit vectors $e_{A}, e_{B}, e_{C}$, denote the angle between $e_{B}, e_{C}$ by $A$, similar notations for $B, C$. Let $e$ be any vector, then

$$
|e|^{2}=\sum_{A} \frac{-\cos A}{\sin B \sin C}\left(e_{A} \cdot e\right)^{2} .
$$

Proof of Theorem 3.1. Let $v$ be a parallel tangent vector field on $\Delta$, then $P_{i}(\omega)(v)$ are constants for $i=1,2 . P_{1}(\omega)(v)=\frac{1}{|\Delta|} \int_{\Delta} \omega(v)$, while for $v$ parallel to one of the edges of $\Delta$ say e, $P_{2}(\omega)(v)=\frac{1}{|e|} \int_{e} \omega(v)$. Let $v_{A}, v_{B}, v_{C}$ be the parallel unit tangent vector field parallel to three edges of $\Delta$ respectively, then by Equation (3.6),

$$
\begin{aligned}
\int_{\Delta}\left|\omega-P_{1}(\omega)\right|^{2} & =\sum_{A} \frac{\cos A}{\sin B \sin C} \int_{\Delta}\left|\omega\left(v_{A}\right)-P_{1}(\omega)\left(v_{A}\right)\right|^{2} \\
& \leq \sum_{A} \frac{\cos A}{\sin B \sin C} \cdot \frac{2 l^{2}}{3} \cdot \int_{\Delta}\left|\nabla \omega\left(v_{A}\right)\right|^{2}=\frac{2 l^{2}}{3} \cdot \int_{\Delta}|\nabla \omega|^{2} .
\end{aligned}
$$

On the other hand, by (3.5),

$$
\int_{\Delta}\left|P_{1}(\omega)\left(v_{A}\right)-P_{2}(\omega)\left(v_{A}\right)\right|^{2} \leq \frac{l^{2}}{8} \int_{\Delta}\left|\nabla \omega\left(v_{A}\right)\right|^{2}
$$

Take the weighted sum and use (3.6), we get

$$
\int_{\Delta}\left|P_{1}(\omega)-P_{2}(\omega)\right|^{2} \leq \frac{l^{2}}{8} \int_{\Delta}|\nabla \omega|^{2}
$$

Apply the inequality $(a+b)^{2} \leq(1+\epsilon) a^{2}+\left(1+\epsilon^{-1}\right) b^{2}$ to (3.7) and (3.8) with $\epsilon=\sqrt{3} / 4$ we get the second inequality of (3.1). 
Lemma 3.5. Let $\omega$ be a harmonic 1-form on $S$ and $-K$ be a lower bound of Gauss curvature of $S$, then

$$
\left\|\nabla_{g} \omega\right\|_{g}^{2} \preceq K\|\omega\|_{g}^{2} .
$$

Proof. From the well-known Bochner-Weitzenbock formula for 1-forms $[4$, p. 28]:

$$
\left(d^{*} d+d d^{*}\right) \omega=\nabla_{g}^{*} \nabla_{g} \omega+\operatorname{Ric} \omega .
$$

For Riemann surfaces, the Ricci operator is the multiplication by Gauss curvature. When $\omega$ is a harmonic 1-form, the left-hand side of above formula vanish; taking inner product with $\omega$ and integrating over $S$, the lemma is then proved.

Combining (2.4), (3.1) and (3.9), we get for $g$-harmonic $\omega$,

$$
\left\|\omega-P_{i} \omega\right\| \preceq(\sqrt{K} L+M)\|\omega\|, \quad i=1,2,
$$

where $M$ is an upper bound of $M(f) l(f)^{2}$ for all $f$, and $L$ is an upper bound of $l(f)$ for all $f$.

Theorem 3.6. Let $\omega$ be a harmonic 1-form on $S$ under metric g, and $N$ is a polygonal surface close to $S$ in the sense of (2.1). Then we have

$$
\left\|\omega-P_{H}\left(P_{2}(\omega)\right)\right\|_{g} \preceq(\sqrt{M}+\sqrt{K} L)\|\omega\| .
$$

Proof. Since $\|\cdot\|_{g}$ and $\|\cdot\|$ are equivalent metrics by (2.1) and (2.2), it is enough to prove for $\|\cdot\|_{g}$ on LHS. $P_{H} P_{2} \omega$ are in the same cohomology class as $\omega$, since $\omega$ is $g$-harmonic,

$$
\begin{aligned}
\left\|\omega-P_{H} P_{2} \omega\right\|_{g}^{2}= & \left\|P_{H} P_{2} \omega\right\|_{g}^{2}-\|\omega\|_{g}^{2} \\
= & \left(\left\|P_{H} P_{2} \omega\right\|_{g}^{2}-\left\|P_{H} P_{2} \omega\right\|^{2}\right)+\left(\left\|P_{H} P_{2} \omega\right\|^{2}-\left\|P_{2} \omega\right\|^{2}\right) \\
& +\left(\left\|P_{2} \omega\right\|^{2}-\left\|P_{2} \omega\right\|_{g}^{2}\right)+\left(\left\|P_{2} \omega\right\|_{g}^{2}-\|\omega\|_{g}^{2}\right) .
\end{aligned}
$$

In Equation (3.13), the first term and the third term can be bounded by $M\|\omega\|^{2}$, applying inequality (2.2). The second term is negative since $P_{H} P_{2} \omega$ is minimal in $L^{2}$ norm among discrete 1-form in the same cohomology class. The last term is bounded by $(\sqrt{K} L+M)^{2}\|\omega\|^{2}$ as in (3.10). The theorem now follows. 
Corollary 3.7. With the same notations, for harmonic 1-form $\omega$, we have

$$
\left\|\omega-P_{H} P_{1} \omega\right\| \preceq(\sqrt{M}+\sqrt{K} L)\|\omega\| .
$$

A smooth holomorphic 1-form is defined to be $\omega+\sqrt{-1} J_{g} \omega$. A natural discrete approximation of this is by $P_{H} P_{2} \omega+\sqrt{-1} P_{H} J P_{H} P_{2} \omega$. The following corollary estimates error of discrete holomorphic 1-forms.

Corollary 3.8. With the same notation as in Theorem 3.6, for harmonic 1-form $\omega$, we have

$$
\left\|J_{g} \omega-P_{H} J P_{H} P_{2} \omega\right\| \preceq(\sqrt{M}+\sqrt{K} L)\|\omega\| .
$$

Proof. Notice that $P_{H}=P_{H} P_{1}$, then

$$
\begin{aligned}
\left\|J_{g} \omega-P_{H} J P_{H} P_{2} \omega\right\| \leq & \left\|\left(J_{g}-P_{H} P_{1} J_{g}\right) \omega\right\|+\left\|P_{H} J_{g} \omega-P_{H} J \omega\right\| \\
& +\left\|P_{H} J \omega-P_{H} J P_{H} P_{2} \omega\right\| .
\end{aligned}
$$

$J_{g} \omega$ is harmonic, so the first term of RHS of (3.16) is bounded by RHS of (3.15) from Theorem 3.6, the second term is bounded by $M\|\omega\|$ from (2.3), the last term is bounded by RHS of (3.15) also from Theorem 3.6.

The period matrix of Riemann surface $S$ is computed by integrating a basis of holomorphic 1-forms over a set of homology basis. Equivalently by Poincaré duality, it equals the matrix of pairing a basis of holomorphic 1-forms with a basis of harmonic 1-forms or even closed 1-forms in the same cohomology class. From the $L^{2}$ estimate of error of holomorphic 1-forms, one easily obtains the following corollary.

Corollary 3.9. The period matrix computed by integrating discrete holomorphic 1-forms over homological basis differs from smooth period matrix by an error bounded by $C(\sqrt{M}+\sqrt{K} L)$, where $C$ is some constant independent of the polygonal approximation surface $N$, but surely depends on geometric property of $S$.

In practice, constant $C$ in the above corollary equals the maximal $L^{2}$ norm of closed discrete 1-forms dual to a set of homological basis of $S$. 


\section{Conclusion}

This paper describes quantitatively the error in computing discrete harmonic or holomorphic 1-forms, the measurement $\sqrt{M}+\sqrt{K} L$ indicates how one should refine a triangulation to compute efficiently.

\section{Acknowledgment}

The author is partially supported by National Natural Science Foundation of China (No. 60503067).

\section{References}

[1] D. Gilbarg and N. S. Trudinger, Elliptic Partial Differential Equations of Second Order, Springer-Verlag, 1983.

[2] X. Gu and S.-T. Yau, Computing Conformal Structures of Surfaces, Commun. Inform. Sys. 2 (2002), no. 2, 121-146.

[3] K. Hilderbrandt, K. Polthier and M. Wardetzky, On the Convergence of Metric and Geometric Properties of Polyhedral Surfaces, ZIB Report 05-24 (April 2005).

[4] J. Roe, Elliptic Operators, Topology and Asymptotic Methods, Longman Scientific \& Technical, 1988.

Center of Mathematical Sciences

ZHEJIANG UNIVERSITY

HANGHZHOU 310027

People's Republic of China

E-mail address: luowei@cms.zju.edu.cn

Received March 15, 2006 